\title{
Polymer collapse and crystallization in bond fluctuation models
}

\author{
Peter Grassberger ${ }^{1,2}$ \\ ${ }^{1}$ JSC, FZ Jülich, D-52425 Jülich, Germany \\ ${ }^{2}$ Max Planck Institute for the Physics of Complex Systems, \\ Nöthnitzer Strasse 38, D-01187 Dresden, Germany
}

(Dated: June 9, 2022)

\begin{abstract}
While the $\Theta$-collapse of single long polymers in bad solvents is usually a continuous (tri-critical) phase transition, there are exceptions where it is preempted by a discontinuous crystallization (liquid $\leftrightarrow$ solid) transition. For a version of the bond-fluctuation model (a model where monomers are represented as $2 \times 2 \times 2$ cubes, and bonds can have lengths between 2 and $\sqrt{10}$ ) it was recently shown by F. Rampf et al. that there exist distinct collapse and crystallization transitions for long but finite chains. But as the chain length goes to infinity, both transition temperatures converge to the same $T^{*}$, i.e. infinitely long polymers collapse immediately into a solid state. We explain this by the observation that polymers crystallize in the Rampf et al. model into a non-trivial cubic crystal structure (the 'A15' or ' $\mathrm{Cr}_{3} \mathrm{Si}^{\prime}$ ' Frank-Kasper structure) which has many degenerate ground states and, as a consequence, Bloch walls. If one controlls the polymer growth such that only one ground state is populated and Bloch walls are completely avoided, the liquid-solid transition is a smooth cross-over without any sharp transition at all.
\end{abstract}

In spite of having been studied for several decades, the phase transitions of a single long polymer in a bad solvent are still a subject of active research with occasional big surprises. When temperature is lowered, most polymers undergo a $\Theta$-collapse, which is typically a continuous (tri-critical) phase transition. At the transition point, repulsive entropic effects and attractive energetic forces would cancel exactly for an infinitely long chain. For finite chain length $N$ theory [1, 2] predicts logarithmic corrections which are also seen in simulations 24 and in experiments [5, and which also affect the unmixing transition of long polymers [6. Qualitatively, the $\Theta$-transition resembles a gas-liquid transition, with the open coil resembling the gas and the dense globule being analogous to a liquid. At even worse solvent conditions, in many models (e.g. in off-lattice polymers with Lennard-Jones interactions between monomers [7]) there occurs a second 'liquid-solid' transition that is similar to crystallization. Since it is a discontinuous transition, its properties are usually not universal and are not described by perturbative renormalization group methods.

But there are well known cases where this $\Theta$-collapse is preempted by a discontinuous 'freezing' or crystallization transition. One example is semi-stiff polymers 8], another is provided by (off-lattice) monomers with hard core repulsion and attractive interactions which extend only very little beyond the core [9, 10. Still another is the cooperative one-step collapse of some proteins 11

A very surprising behavior which falls between these two possibilities $-\Theta$-collapse with subsequent liquid-solid transition, and immediate gas-solid freezing - was observed by Rampf et al. 12 16. In their model, the specific heats of finite chains show rounded peaks at distinct temperatures $T_{\text {crys }}(N)<T_{\Theta}(N)$, but as the chain length $N$ diverges,

$$
\lim _{N \rightarrow \infty} T_{\text {crys }}(N)=\lim _{N \rightarrow \infty} T_{\Theta}(N)=T^{*} .
$$

Thus, while finite chains show the expected two distinct transitions, infinitely long polymers collpase in a single "hybrid" transition into their ground state [17.

In [9, 10] it was conjectured that this is connected to the range of interactions in this model, since the phenomenon disappeared when also more distant monomer pairs are included in the interaction. But it is not clear what would be the detailed mechanism for that. In the present paper, we propose a different explanation which is based on the fact that discontinuous phase transitions arise typically if there is a large gap between the (few) low energy states that dominate the low- $T$ phase and the bulk of states at higher energies. This is of course also the reason why there is a first order collapse in hard-core polymer models with very short range attraction: The shorter the range of the attractive potential, be bigger will be the effect of a single displaced monomer, as it will cause many monomer distances to be out of the interaction range. In the Rampf et al. model, the energetic distance between ground and excited states is, in contrast, dominated by topological defects similar to Bloch walls in magnetic systems that arise from a spontaneously broken symmetry.

We verify this conjecture by studying also a modified model where all such topological defects are completely eliminated, by constraining all configurations to be in the same ergodic component. This modified model has of course the same ground state energy, but it has a dramatically changed phase diagram: There is a standard $\Theta$ transition, but no crystallization transition at all. Instead, the transition from a disordered "liquid" to the densely packed ground state is a continuous cross-over. A similar behavior seems to prevail for interacting selfavoiding walks on the simple cubic lattice, although the interpretation there is controversial [18. In the very careful studies of [18, clear peaks in the specific heat were observed which obviously are related to some crystallization phenomena. But all indications point to the fact that they are not associated with bulk crystallization, 
but with changes in the geometric shapes and surface properties of clusters which are already completely compact and ordered in their bulk.

The model used by Rampf et al. is the bond fluctuation model (BFM) of Carmesin et al. [19], augmented by an ansatz for the interactions. The BFM lives on a simple cubic lattice. Monomers are represented by $2 \times 2 \times 2$ cubes, i.e. if site $\mathbf{x}=(x, y, z)$ is occupied by a monomer, all sites with Euclidean distance $<2$ are excluded. These monomers are linked by bonds with integer coordinate components and length $2 \leq l \leq \sqrt{10}$. Thus the set of all bond vectors is made up by the vectors $(2,0,0),(2,1,0),(2,1,1),(2,2,0),(2,2,1),(3,0,0),(3,1,0)$ and all their rotations and reflections (altogether 108 vectors).

The solvent is not treated explicitly, but implicitly by assuming an attractive (negative) contact energy for any pair of monomers that touches each other along part of their surface (line and corner contacts do not contribute to this energy). Notice that two monomers can touch on their full $2 \times 2$ sides, or on contact areas of size $2 \times 1$ or $1 \times 1$. If every contact would make a contribution $-\epsilon \mathcal{A}$ to the total energy (where $1 \leq \mathcal{A} \leq 4$ is the contact area), the total energy of compact configuration (i.e., of a tiling of 3 -d space by $2 \times 2 \times 2$ cubes) would be $-12 \epsilon$ per monomer, independent of the way how space is tiled.

This is, however, not the interaction model chosen in [12. There it was assumed that every touching pair (including those which are joined by a bond) contributes $-\epsilon$ to the energy, independent of the area of contact. Thus for achieving minimal energy it is preferred to avoid fullside contacts and to maximize the number of surfaces which are in partial contact. In the following we shall assume that the minimal energy configuration is indeed a tiling, i.e. the monomers occupy space densely. We have no rigorous proof for this, but it is fully compatible with the simulations. As shown in [20], there exist nine inequivalent tilings of space by cubes of the same size. All of them except one are made up by perfect layers. The one not made up by perfect layers is the one with the largest number of contacts. It is periodic, and every cube in it has in average $27 / 2$ neighbors in contact (the next best tiling is by layers consisting of simple square lattices, each layer shifted by by one unit. In this tiling, each cube has 12 neighbors).

Indeed, this optimal tiling is nothing but the A15 (or $\mathrm{Cr}_{3} \mathrm{Si}$ ) Fank-Kasper structure [21. Usually, in this structure particles are represented not by cubes but by spheres, and their centers are then slightly displaced with respect to the ideal lattice positions. In nature, this crystal structure is realized for binary alloys of composition $\mathrm{A}_{3} \mathrm{~B}$ where the $\mathrm{A}$ atoms are slightly larger than the $\mathrm{B}$ atoms (as far as I am aware of, the fact that the A15 structure corresponds to a cube tiling was not known before). All alloys with A15 structure are very brittle, due to the absence of easy gliding planes when seen as packings of cubes. The A atoms have coordination number 14 , while the $\mathrm{B}$ atoms have coordination number 12 , giv- ing rise to the average $27 / 2$ mentione above. Seen as a cubic crystal structure, the basic unit cell has size $4 \times 4 \times 4$ and contains 8 atoms (monomers) at the sites

$$
\begin{aligned}
& (0,0,0),(1,1,2),(1,2,0),(1,3,2), \\
& (2,0,0),(3,0,2),(3,2,1),(3,2,3) .
\end{aligned}
$$

For any perfect arrangement of atoms in such a structure there exist many other arrangements which are just shifted copies of it. Thus this structure, seen as a ground state of a statistical mechanics model, is highly degenerate. If we prepare a big system such that it is in one of these ground states in some region of space of characteristic size $L$ and in another ground state somewhere else, there must be "Bloch walls" between them. These are toplological defects with energies $\propto L^{2}$. It is these Bloch walls which are responsible for the discontinuity and high temperature of the freezing transition.

In contrast to [12 16, where Markov chain Monte Carlo methods were used, we used PERM [4] and "flat PERM" 22]. PERM is a growth algorithm with importance sampling and re-sampling, i.e. chains are grown in a biased way, with the bias compensated by weight factors. On the basis of weights of partially grown chains, the "population" of chains is controlled, similarly as in genetic models, by pruning and cloning. In the original PERM algorithm the growth is controlled by comparing the weight of the current configuration with the estimated partition sum, i.e. with the average weight of all previous configurations with the same length. In flat PERM it is compared with the average weight of those previous configurations that have the same length and energy. Both versions of the algorithm work perfectly, if the current weights of partially grown chains are good indicators of their final weights when the growth is finished. This is true for polymers at temperatures above $T_{\Theta}$, and even more so at $T_{\Theta}$, where its success is spectacular [4]. But it deteriorates quickly, if growth conditions change much during the growth and configurations which finally would be very good are pruned away at early stages. This happens typically in first order phase transitions. While Markov chain Monte Carlo methods are based on the concept of changing complete configurations to increase their Boltzmann weight, in PERM no configuration can be changed once it has been created.

In addition, we tried also another version called "new PERM" (nPERM) 23]. All three methods gave very similar results. Typical "good" results are shown in Fig. 1. There we present estimates of the average squared endto-end distances, plotted against $N$ for several temperatures near $T_{\Theta}$. At $T=T_{\Theta}$ one expects

$$
R_{\text {end-end }}^{2} \propto N
$$

up to logarithmic corrections. Although the leading corrections are known theoretically [1, 2, it is well known that they do not quantitatively describe the behavior at presently reachable chain lengths 2 4. Nevertheless we clearly see deviations from Eq. (3) which qualitatively 


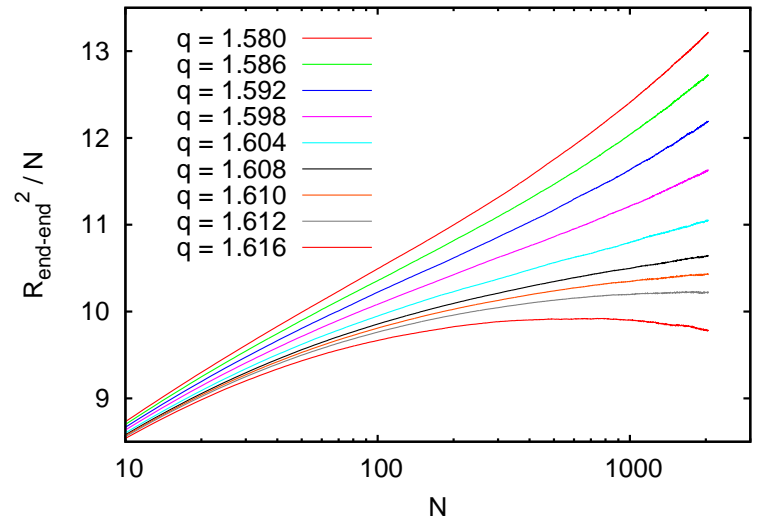

FIG. 1. (Color online) Semi-logarithmic plot of $N^{-1}$ times the average squared end-to-end distances against chain length $N$. Instead of temperature, we use the Botzmann factor $q=$ $\exp \left(\epsilon / k_{B} T\right)$ to label each curve. At $T=T_{\Theta}$ one expects a horizontal curve up to logarithmic corrections.

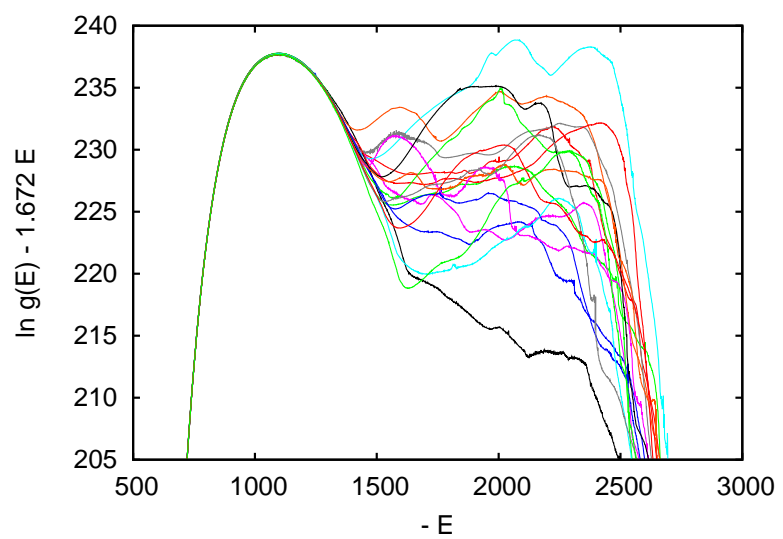

FIG. 2. (Color online) Logarithms of the density of states $g(E)$, plotted against tne energy $E$. In order to see more clearly the two humps expected from the fact that there is a first order freezing transition, we actually plotted $\log g(E)-$ 1.672E. Each curve is from a different flat PERM run with different random number seeds and using slightly different versions of the algorithm, thus ideally these curves should collapse.

agree with the theoretical predictions. We fully confirm the estimates for $T_{\Theta}$ given in [12 16, but due to the much longer chains and higher statisitics we can give much smaller error bars in spite of the theoretical uncertainty:

$$
T_{\Theta}=2.103(10) .
$$

One advantage of PERM is that it allows to estimate absolute values of partition sums and of the density of

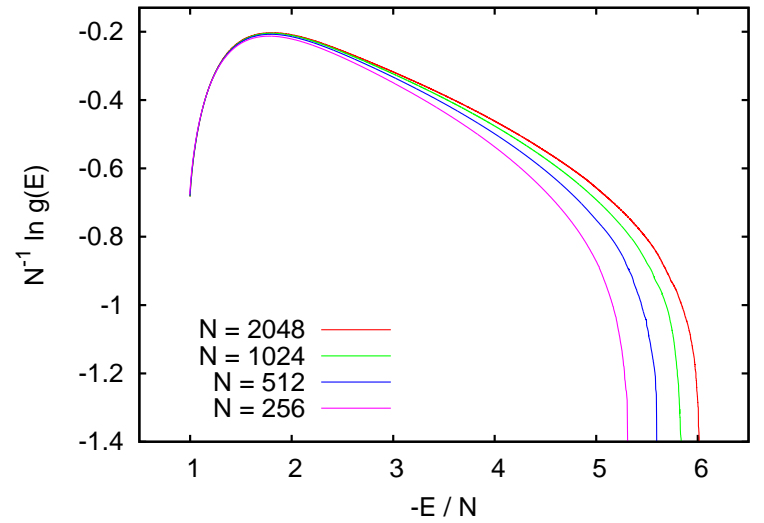

FIG. 3. (Color online) Logarithms of the density of states $g(E)$ per monomer, plotted against the energy per monomer, for the modified model restricted to the sites in a perfect crystal.

states $g(E)$, where $E$ is the total energy. Using $\epsilon=1$ in the following, $|E|$ is just the number of contacts. Indeed, these estimates are needed to controll the population growth, so estimating them is an essential part of the algorithm. In Fig. 2 we show $\ln g(E)+1.672 E$ at fixed $N=512$ against $E$ for several runs. Notice that 1.672 is the value of the Boltzmann factor per contact at the effective freezing temperature according to [12]. Thus we expect the curve to be double-humped with both maxima having roughly the same height. Each run used different random number generator seeds and different minor parameters in the algorithm, and ran for about one week of CPU time on a fast PC. We see very good results for large $E$ (i.e. for energies relevant at $T \geq T_{\Theta}$ ), but a complete failure at energies which, according to [12, dominate the crystallized phase. The only positive statement these simulations allow to draw is that there is presumably really a first order transition, since otherwise the failure would not be expected to be as dramatic. Similar plots were obtained for all $128 \leq N \leq 1024$.

Let us now finally look at simulations of the constrained model, where we allowed monomers to be placed only at one of the positions given in Eq. (2) (Fig. 3). This time we show data for different values of $N$, up to $N=2048$. The data now look absolutely clean. Moreover, we obtained good data down to energies which are close to the expected ground state energy $E_{\min }=$ $-6.75 N+O\left(N^{2 / 3}\right.$ (see Fig. 4). It could of course be that these results are spurious, and that the simulations just missed those configurations which would have created troubles. We consider this as highly unlikely and take Fig. 3 as an indication that there is really no crystallization transition. Instead, when temperature is lowered, holes in the configuration are gradually filled up until a compact configuration is reached. Indeed, Fig. 3 looks 


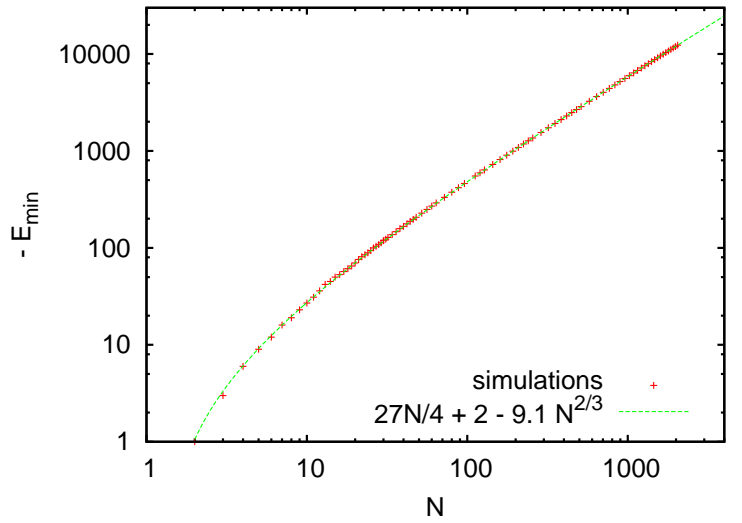

FIG. 4. (Color online) Energy minima reached in simulations of the modified model for $N=2048$. The continuous curve is a fit with the predicted bulk term $27 / 4 N$ and a surface term $\propto N^{2 / 3}$. much better than analogous plots for the simple cubic lattice. There we of course also have no toplogocal defects, but the much lower coordination number implies that the growth is much more often blocked at low temperatures. For the fcc lattice, however, where coordination number is 12 , we again found very clean results.

In summary, we have shown that the very strong discontinuous crystallization transition in the polymer model of 12 is due to spontaneously broken translational symmetry in its low energy state. Similar discontinuous transitions are expected (and found) in continuum models, if the polymer can freeze into a periodic monomer configuration. We do not expect any first order transition if the low energy state is glassy (such as in a Lennard-Jones homopolymer with bond lengths incommensurate with the Lennard-Jones radius) and in lattice models without spontaneous symmetry breaking, such as e.g. the simple cubic lattice. The reason why crystallization is qualitatively different in the bond fluctuation model of [9, 10] is primarily not because of the increased average range of interactions, but because of the changed structure of the ground state and low energy excitiations.

For very helpful correspondence I thank W. Paul, P. Paufler, H. Chaté and M. Dutour Sikiric.
[1] B. Duplantier, Journal of Chemical Physics 86, 4233 (1987).

[2] J. Hager and L. Schäfer, Physical Review E 60, 2071 (1999).

[3] P. Grassberger and R. Hegger, Journal of Chemical Physics 102, 6881 (1995).

[4] P. Grassberger, Physical Review E 56, 3682 (1997).

[5] A. Boothroyd, A. Rennie, C. Boothroyd, and L. Fetters, Physical Review Letters 69, 426 (1992).

[6] H. Frauenkron and P. Grassberger, Journal of Chemical Physics 107, 9599 (1997).

[7] S. Schnabel, M. Bachmann, and W. Janke, J. Chem. Phys. 131, 124904 (2009).

[8] U. Bastolla and P. Grassberger, Journal of Statistical Physics 89, 1061 (1997).

[9] M. P. Taylor, W. Paul, and K. Binder, Journal of Chemical Physics 131, 114907 (2009).

[10] M. P. Taylor, W. Paul, and K. Binder, Physical Review E 79, 050801 (2009).

[11] Notice that only monomer positions, not the the location of bonds is frozen out in homopolymer crystallization, while also the latter are frozen out in protein folding. Thus the often cited analogy between protein folding and the crystallization of homopolymers should not be stretched too far, as the cooperativity of protein folding is entirely due to their heterogeneity.

[12] F. Rampf, W. Paul, and K. Binder, Europhysics Letters 70, 628 (2005).

[13] F. Rampf, K. Binder, and W. Paul, Journal of Polymer Science Part B: Polymer Physics 44, 2542 (2006).

[14] W. Paul, T. Strauch, F. Rampf, and K. Binder, Physical Review E 75, 060801 (2007).

[15] K. Binder, J. Baschnagel, M. Müller, W. Paul, and
F. Rampf, in Macromolecular symposia, Vol. 237 (Wiley Online Library, 2006) pp. 128-138.

[16] K. Binder, W. Paul, T. Strauch, F. Rampf, V. Ivanov, and J. Luettmer-Strathmann, Journal of Physics: Condensed Matter 20, 494215 (2008).

[17] Hybrid transitions that combine aspects of both first and second order have recently been found also in other models 24 28, but there seems to be no closer connection.

[18] T. Vogel, M. Bachmann, and W. Janke, Physical Review E 76, 061803 (2007).

[19] I. Carmesin and K. Kremer, Macromolecules 21, 2819 (1988).

[20] M. Dutour Sikiric, Y. Itoh, and A. Poyarkov, "Torus cube tilings," http://drobilica.irb.hr/ mathieu/ Presentations/CubeTiling.pdf/ [accessed 24-May2013].

[21] M. De Graef and M. E. McHenry, Structure of materials: an introduction to crystallography, diffraction and symmetry, 2nd ed. (Cambridge University Press, 2012).

[22] T. Prellberg and J. Krawczyk, Physical Review Letters 92, 120602 (2004).

[23] H.-P. Hsu, V. Mehra, W. Nadler, and P. Grassberger, Journal of Chemical Physics 118, 444 (2003).

[24] M. S. Causo, B. Coluzzi, and P. Grassberger, Physical Review E 62, 3958 (2000).

[25] S. N. Dorogovtsev, A. Goltsev, and J. F. F. Mendes, Physical Review Letters 96, 040601 (2006).

[26] J. Schwarz, A. J. Liu, and L. Chayes, EPL (Europhysics Letters) 73, 560 (2006).

[27] A. Goltsev, F. De Abreu, S. Dorogovtsev, and J. Mendes, Physical Review E 81, 061921 (2010).

[28] G. Bizhani, M. Paczuski, and P. Grassberger, Physical Review E 86, 011128 (2012). 